\title{
MÉTODOS DE VALORACIÓN JURÍDICOS, CONTABLES Y ECONÓMICO-FINANCIEROS APLICABLES EN UNA FUSION DE SOCIEDADES ANÓNIMAS
}

\author{
JENNIFER ISABEL ARROYO CHACÓN \\ Contraloría General de República de Costa Rica \\ prof.jenniferarroyo@yahoo.com
}

\section{RESUMEN}

Un mundo cada vez más globalizado como el actual, en donde la apertura de mercados permite el ingreso de nuevos competidores, lleva a los negocios locales de un país a buscar alternativas para enfrentar la competencia; o bien, buscar alternativas para el crecimiento y dominio del mercado. Entre dichas alternativas surge la fusión de sociedades anónimas como una manera en que dos o más empresas dedicadas a actividades mercantiles similares se unen, ya sea para hacerle frente a un competidor fuerte, en virtud, de que si quedaren separadas no podrían resistir mucho tiempo ante las estrategias para dominar el mercado del nuevo competidor; o también, para lograr el control de una importante porción del mercado. El tema de las fusiones de sociedades resulta bastante complejo; ya que en ella intervienen muchas disciplinas que pueden definir el éxito o fracaso de la negociación; no obstante, el presente análisis se enfoca en tres áreas básicas que son: Ia jurídica, la económica y la contable. La perspectiva jurídica se abarca desde el método del Legal Due Diligencie, mientras que la esfera contable se estudia a través de la NIIF 3: Combinación de Negocios y la NIC 7: Estados de Flujos de Efectivo. Finalmente, se analizan los distintos métodos financieros que se utilizan para valorar empresas, con especial mención en el Flujo de Caja Libre (Cash Flow), como el método financiero más conveniente para una fusión de sociedades anónimas. De esta manera, el presente artículo pretende desarrollar las principales normas de los ámbitos jurídico, económico y contable que se aplican al momento de realizar una fusión de sociedades y a fin de determinar el valor de la nueva sociedad originada por la combinación de los activos y pasivos de las sociedades predecesoras.

PALABRAS CLAVE: FUSIÓN DE SOCIEDADES ANÓNIMAS, MÉTODOS DE VALORACIÓN, LEGAL DUE DILIGENCE, NIIF 3, NIC 7, FLUJO DE CAJA LIBRE.

\begin{abstract}
An increasingly globalized world -where the opening of markets allows the entrance of new competitors - forces local businesses to find alternatives to face competition or to look for new ideas for growth and market control. Between these options, corporation merging becomes an alternative for two or more companies to engage in similar business activities either to face a strong competitor-since, separated, they could not endure for too long the strategies for market control of a stronger new competitor-or to gain control of a major market share. Corporate mergers are quite complex, since it involves many disciplines that determine the success or failure of the negotiation; this article analyzes three basic disciplines: law, economy and accounting. The legal perspective is analyzed with the Legal Due Diligence method, while accounting is studied through IFRS 3: Business Combinations and IAS 7: Cash Flow Statements. Finally, various financing methods are mentioned as used to value companies, especially Free Cash Flow (Cash Flow) as the most suitable financing method for corporative merging. This article explores the main legal, economic and accounting rules that apply for a merger of companies and determine the value of the new society resulted by the combination of assets and liabilities of previous companies.
\end{abstract}


KEYWORDS: MERGER OF CORPORATIONS, VALUATION METHODS, LEGAL DUE DILIGENCIE, IFRS 3, IAS 7, FREE CASH FLOW.

\section{INTRODUCCIÓN}

Ante la apertura de mercados, derivados de tratados de libre comercio, inversión extranjera y demás fenómenos comerciales; se incrementa la competencia en el país y, por lo tanto, la fusión de sociedades anónimas se muestra como estrategia de integración, aprovechando el principio de sinergia, a fin de unir fuerzas y hacerle frente a competidores grandes.

Ahora bien, la decisión de fusionarse resulta sumamente relevante y posee fuertes impactos en las empresas participantes, que podrían o bien ser la alternativa idónea para mantener la empresa en el mercado; o por el contrario condenar a la empresa al fracaso.

Esta decisión debe ser minuciosamente estudiada desde todos sus aspectos, pues en ocasiones se dejan áreas importantes sin analizar y los empresarios se sorprenden al ver que la empresa no era tan rentable como se esperaba y ello conlleva al fracaso de la fusión con las consiguientes pérdidas económicas y de oportunidad para el negocio.

En consecuencia, para llevar a cabo una fusión no resulta suficiente con revisar los aspectos económicos-financieros, sino que también hay que evaluar la situación jurídica y las implicaciones contables de este negocio.

Ello, por cuanto la fusión de una sociedad posee razones económicas para las empresas y un impacto en la economía nacional, pero también está regulada por un marco normativo que debe respetar, y establece no solo el proceso a seguir para configurar una fusión, sino también las implicaciones en los derechos de terceros involucrados en la transacción.
Asimismo, inciden las normas contables al momento de contabilizar la fusión y determinar el valor de las acciones de la nueva sociedad originada por la combinación de los activos y pasivos de las dos sociedades que le dieron origen.

De esta manera, el presente artículo pretende analizar la convergencia de las áreas jurídicas, contables y económico-financieras que se aplican en una fusión de sociedades anónimas (ver figura 1).

FIGURA 1

\section{Convergencia áreas jurídicas, contables y económico y financiero}

Contable

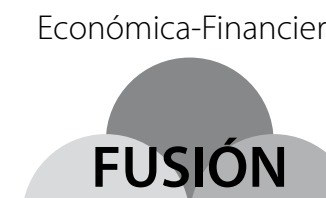

\section{Concepto y tipos de fusión de sociedades}

El numeral 220 del Código de Comercio Costarricense define la fusión de sociedades anónimas como la unión de dos o más sociedades para formar una nueva.

Dicho texto indica:

ARTíCULO 220.- Hay fusión de sociedades cuando dos o más de ellas se integran para formar una sola. Las sociedades constituyentes cesarán en el ejercicio de su personalidad jurídica individual cuando de la fusión de las mismas resulte una nueva. Si la fusión se produce por absorción, deberá modificarse la escritura social de la sociedad prevaleciente, si fuere del caso (Asamblea Legislativa, 1964). 
Por su parte, el Tribunal Contencioso Administrativo, en su sentencia número 00149-01 del 25 de mayo de 2001, ha definido la "fusión" como:

IV.- En doctrina, se habla de fusión para aludir al fenómeno jurídico de mezcla de empresas sociales y se distinguen dos tipos: 1 . - fusión por integración, cuando dos o más sociedades se disuelven, se unen sin liquidarse y constituyen una nueva sociedad y 2. - fusión por absorción o incorporación, que se da cuando la sociedad absorbida se incorpora a otra, y sus socios reciben la correspondiente participación social en la sociedad prevaleciente. En todo caso, la nueva sociedad o la prevaleciente adquiere la titularidad de la totalidad de los derechos y obligaciones de las sociedades disueltas, al producirse la transferencia de patrimonio.

Nótese que tanto en el numeral como en la jurisprudencia citada se establecen los dos tipos de fusiones que existen:

a) Fusión por Consolidación o Fusión por Integración: cuando dos o más empresas se unen para formar una nueva y desaparecen las anteriores.

\section{Empresa A + Empresa B $=$ Empresa C}

b) Fusión Estatutaria o Fusión por Incorporación: cuando una empresa grande absorbe a otra empresa generalmente más pequeña, en donde la empresa grande sobrevive y se denomina "sobreviviente"; o bien, "adquirente" pues es la empresa que compra, y la empresa pequeña desaparece.

\section{Empresa $\mathrm{A}+$ Empresa $\mathrm{B}=$ Empresa $\mathrm{A}$}

Igualmente, según el Instituto Mexicano de Contadores Públicos (2001), la fusión de socie- dades desde la perspectiva económica puede clasificarse de la siguiente manera:

a) Fusión Vertical: cuando la fusión se da con el fin de hacer una integración con un eslabón dentro del proceso productivo, sea hacia delante o hacia atrás, es decir, cuando la empresa busca ser su propio cliente o su propio proveedor.

b) Fusión Horizontal: cuando se fusionan dos empresas que poseen actividades comerciales similares en la misma zona.

c) Fusión Conglomerada: cuando se fusionan empresas que poseen actividades lucrativas distintas o en sectores diferentes. Este tipo de fusión se subdivide en tres supuestos que son:

i. Extensión del producto: se da entre empresas que manejan sistemas de producción o distribución similares y cuyos productos no compiten directamente entre sí.

ii. Extensión del mercado: se lleva a cabo entre empresas que fabrican productos similares, pero en distintos mercados.

iii. Fusión sin relación: cuando se fusionan empresas que no tienen relación alguna y esta adquisición se realiza con la finalidad de entrar a nuevos mercados, o por alguna otra de las razones mencionadas anteriormente.

Toda fusión se caracteriza por combinar los activos y pasivos de dos o más sociedades, generando la creación de una nueva sociedad a partir de los aportes de sus predecesoras.

Asimismo, en la fusión siempre va a desaparecer al menos una sociedad, como es el caso de la fusión por incorporación o estatutaria; o bien, ambas, como en la fusión por consolidación o integración. 
Ahora bien, en el marco jurídico costarricense las normas que regulan esta figura en el Código de Comercio son escasas y únicamente se refieren al trámite para publicitar e inscribir la fusión.

El trámite establecido en el Código de Comercio para llevar a cabo la fusión de sociedades consta de tres partes:

\section{Acuerdos Internos de fusión en cada sociedad}

El artículo 221 señala que aquellas sociedades que pretendan fusionarse deberán elaborar un proyecto de acuerdo, que debe ser aprobado en el seno de cada una de las empresas que participan en el negocio, haciendo constar la sesión de aprobación del acuerdo en los libros de actas de cada sociedad y por medio de escritura notarial.

Dicho numeral señala:

ARTíCULO 221.- Los representantes legales de cada una de las sociedades que intenten fusionarse prepararán un proyecto de acuerdo que firmarán y en el cual se harán constar los términos y condiciones de la fusión, el modo de efectuarla y cualesquiera otros hechos y circunstancias que sean necesarios de acuerdo con sus respectivas escrituras sociales. El acuerdo de fusión deberá ser sometido a los socios de cada una de las sociedades constituyentes, en sendas asambleas extraordinarias convocadas al efecto, y deberá ser aprobado por cada sociedad conforme a los requisitos que su escritura social exija para ser modificada y a los establecidos en este Código. Un extracto de la escritura de fusión se publicará por una vez en el Diario Oficial (Asamblea Legislativa, 1964).
Publicación de dichos acuerdos en el periódico oficial

Una vez aprobados los acuerdos de fusión en el seno de la asamblea general de cada una de las sociedades que participan, resulta necesario hacer una publicación de estos acuerdos en el periódico oficial, La Gaceta.

Lo anterior busca darle publicidad a este tipo de operaciones que, por su complejidad e importancia, se mantienen en secreto; y a su vez, amparar los derechos de los terceros vinculados con la empresa que pudieren salir afectados con la fusión a fin de que puedan oponerse y que sus derechos sean tutelados.

\section{Inscripción en el Registro}

Finalmente, la fusión debe inscribirse en el Registro Nacional, en el apartado de personas jurídicas para informar a cualquier persona que tenga interés en las sociedades participantes que ha acontecido un proceso de fusión.

Las anteriores dos etapas, se encuentran reguladas en el numeral 222 del Código de Comercio, donde se establece la obligación de publicar e inscribir la fusión en el Registro Nacional.

Asimismo, prevé la posibilidad de que un tercero que se considere afectado por la fusión pueda oponerse a esta y la fusión no tendrá efectos hasta que no se tutelen los derechos de la parte afectada.

ARTÍCULO 222.- La fusión tendrá efecto un mes después de la publicación y una vez inscrita en la Sección Mercantil del Registro Público.

Dentro de dicho plazo, cualquier interesado podrá oponerse a la fusión, que se suspenderá en ese caso en tanto el interés del opositor no sea garantizado suficientemente, a juicio del Juez que conozca de la demanda. 
Si la sentencia declarare infundada la oposición, la fusión podrá efectuarse tan pronto como aquélla cause ejecutoria (Asamblea Legislativa, 1964).

De esta manera, los aspectos regulados en el ordenamiento jurídico costarricense se vinculan únicamente al proceso de publicidad e inscripción de la fusión, pero también se dejan por fuera otros múltiples elementos relevantes para este proceso, por lo tanto, la legislación resulta omisa en muchos temas vinculados con la figura de la fusión de sociedades y su impacto en la economía nacional.

De lo expuesto se puede obtener como una afirmación importante que la fusión de sociedades anónimas constituye una estrategia válida en el mundo de los negocios, y se encuentra tutelada por el ordenamiento jurídico costarricense.

Igualmente, que durante su realización se integran y convergen diferentes métodos de valoración en los ámbitos jurídicos, contables y económicos financieros, los cuales se pretenden estudiar en los acápites siguientes de este documento (ver Figura 2).

\section{Método Jurídico: Legal Due Diligence}

\section{Concepto}

El Legal Due Diligence se deriva del método del Due Diligence, o "Método de la Debida Diligencia", que tiene su origen en el área de las finanzas corporativas, y que se define como el proceso para estudiar, investigar y evaluar una empresa con el fin de determinar su valor y si es prudente llevar a cabo algún tipo de transacción comercial con ella.

Sobre esta figura se ha dicho lo siguiente:

Un trabajo de Due Diligence consiste en el análisis de investigación de las actividades financieras y operativas de una empresa relacionado con una posible transacción que resultaría en un cambio significativo en la propiedad o composición del capital social de la empresa objetivo.

El papel del trabajo de Due Diligence es fundamental para el proceso de una fusión o adquisición de empresas. Un Due Diligence

FIgURA 2

\section{Fusión de Sociedades}

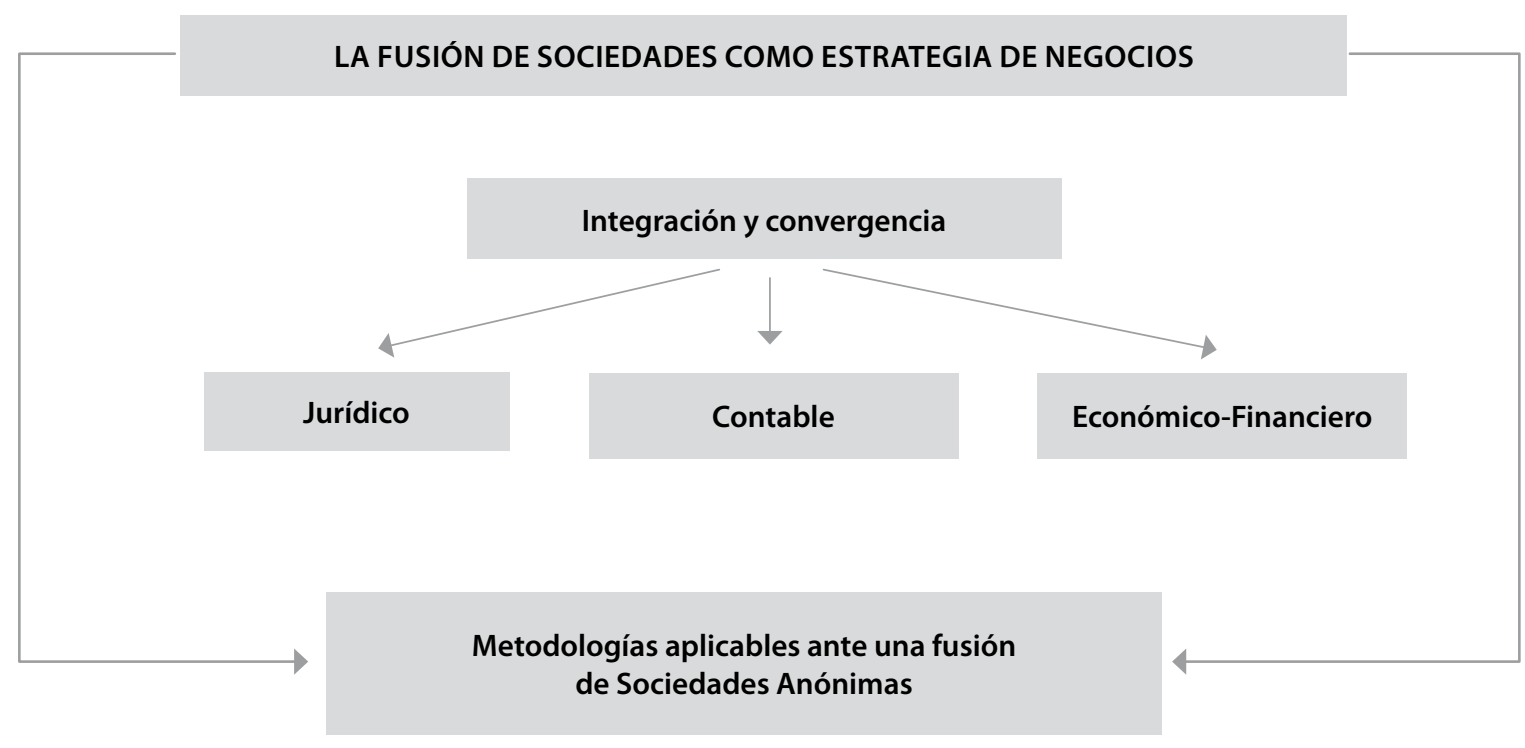

Fuente: Elaboración propia. 
es un proceso que se utiliza para estudiar, investigar y evaluar la oportunidad de realizar un negocio. Se efectúa normalmente justo después de que las partes interesadas han llegado a la conclusión de que el negocio es factible desde el punto de vista financiero y después de que se ha logrado un entendimiento preliminar, pero antes de que se firmen los documentos que legalizan la compra del negocio (López, 2006, pp. 27 y 28).

El Due Diligence es un proceso que se ejecuta cuando las empresas pretenden llevar a cabo una fusión, pero que aún no se ha definido si esta se va a ejecutar, ya que este método suministra información a las partes involucradas para que tomen la decisión final, de fusionarse o no, según lo que más le conviene a sus intereses. Para ello, se requiere de información confidencial de la empresa o empresas involucradas en el negocio, razón por la cual, este se ejecuta con suma confidencialidad y se suscriben acuerdos garantizando la discreción de las partes y profesionales que lo van a ejecutar.

Dentro del método del Due Diligence existen diferentes áreas de trabajo, que realizan una investigación pormenorizada de todos los aspectos importantes de una empresa, tanto en el ámbito financiero, legal y medioambiental con el objetivo de suministrar información completa para la toma de decisiones (Tabla 1).

De las áreas de trabajo mencionadas anteriormente, interesa para efectos del presente documento desarrollar, el análisis fiscal, legal y laboral, que es el que constituye el Legal Due Diligence.

El Legal Due Diligence es el mecanismo mediante el cual, se determina la situación en que se encuentran las sociedades a fusionarse en el cumplimiento de sus obligaciones legales, sea el respeto de las obligaciones fiscales y tributarias y la normativa comercial que se le aplica, así como la situación de las demandas existentes, tanto las planteadas en su contra como aquellas en que funge como actor, y las posibles demandas futuras que existe el riesgo de que se concreten.

La doctrina ha definido esta figura de la siguiente manera:

La due diligence legal es la herramienta que tiene cualquier persona para que, a través de un procedimiento de investigación, se analice e interprete la situación legal de una entidad, según los alcances establecidos.

TABLA 1

\section{Método Due Diligence}

\begin{tabular}{|l|l|}
\hline \multirow{2}{*}{ Análisis financiero } & $\begin{array}{l}\text { 1. Estados financieros } \\
\text { 2. Cuentas }\end{array}$ \\
\hline \multirow{2}{*}{ Análisis fiscal, legal y laboral } & $\begin{array}{l}\text { 3. Proyecciones } \\
\text { 4. Datos históricos }\end{array}$ \\
\hline Análisis medioambiental & $\begin{array}{l}\text { 1. Comprobar el cumplimiento de las obligaciones fiscales y posibles contingencias. } \\
\text { 3. Revisar toda la documentación mercantil y otros aspectos societarios. }\end{array}$ \\
\hline & $\begin{array}{l}\text { Determinar y cuantificar posibles riesgos y verificar el cumplimiento de la normativa apli- } \\
\text { cable en materia de medio ambiente. }\end{array}$ \\
\hline
\end{tabular}

Fuente: Elaboración propia. 
Se desprende del texto que la due diligence legal tiene por finalidad la obtención del mayor conocimiento de la empresa investigada, lo cual se traduce en la identificación de oportunidades y riesgos vinculados con el negocio de la empresa, la identificación de pasivos y contingencias que podrían eventualmente implicar un ajuste al precio de compra o un incremento en las garantías del vendedor del negocio y un mayor nivel de seguridad y tranquilidad para la persona que está interesada en la compra de un negocio (Tajada, 2011, p. 213).

En este análisis se estudia tanto la normativa costarricense aplicable así como posibles conflictos jurídicos por normas de otros países que interfieran con el negocio, tales como el caso de los exportadores, o cuando se poseen importantes socios comerciales en otras latitudes y existen marcos jurídicos diferentes incidiendo en la misma empresa.

Este método suministra información valiosa para el comprador, quien es normalmente el que lo solicita, igualmente, dichos datos son importantes para el vendedor pues le permite conocer mejor su empresa y así posee mejores criterios para determinar su valor.

\section{Importancia}

El Legal Due Diligence suministra información para las partes involucradas, que va más allá del análisis de datos financieros y económicos, que lógicamente se realizan en este tipo de operaciones, tales como demandas planteadas ante los Tribunales de Justicia en contra de la empresa y que impliquen una indemnización importante, o bien, gravámenes sobre los activos objeto de transmisión, sanciones comerciales que posea la empresa, incumplimientos tributarios que pudieran ser sancionados, entre otros.
De tal manera que la información obtenida a través del Legal Due Diligence puede influir al momento de determinar el precio de la negociación, sea para incrementarlo o bien para disminuirlo, dependiendo de la situación encontrada.

Por lo tanto, los beneficios que se pueden esperar de este método son los siguientes:

1) Permite identificar los riesgos de la operación

2) Aporta argumentos a la hora de determinar el valor de la empresa

3) Proporciona información valiosa para la negociación

4) Identifica potenciales sinergias

5) Determina la estructura de la operación.

Fases del proceso del Legal Due Diligence

El Legal Due Diligence está dividido en 4 fases:

1. Fase preliminar: en esta fase, el comprador conforma su equipo de abogados especialistas en distintas áreas, tales como derecho laboral, comercial, tributario, aduanas, propiedad intelectual y cualquier otra especialidad que sea necesaria por la naturaleza de la empresa.

A su vez, el vendedor designa a los profesionales de su empresa que van a atender las peticiones de información del equipo de trabajo del comprador.

En esta fase preliminar hay que tener muy claros una serie de puntos:

- Comprensión del objetivo; quién compra; por qué compra; cómo paga, entre otros.

- Delimitación del alcance del trabajo, para mantener un equilibrio suficiente 
entre el nivel de riesgo, el tiempo, los costes y los recursos de que se dispone.

- Fijación de un calendario de trabajo.

- Aceptación de la propuesta de servicios.

2. Fase de investigación: en esta fase recoge y analiza la información y se procede a realizar el cuestionario preliminar o Check List, donde se incluyen diferentes aspectos, Tajada (2011) cita los siguientes:

- Revisión de aspectos societarios

- Revisión de aspectos contractuales

- Revisión de aspectos relacionados con la propiedad

- Revisión de aspectos vinculados con el derecho administrativo

- Revisión de aspectos laborales

Finalmente, se puede incluir cualquier otra especialidad que se considere relevante para la decisión de compra-venta en virtud de la actividad lucrativa de la empresa.

3. Fase de confirmación de datos: se analiza la información recopilada según la importancia jurídica que se posee, y para ello se suele pactar una serie de reuniones entre vendedor y comprador, y los equipos de cada uno.

4. Emisión del informe de Legal Due Diligence: el informe del Legal Due Diligence recoge toda la información recopilada y analizada en las etapas anteriores. Se estructura de la siguiente manera:

- Introducción: con alcance y razón del trabajo de Legal Due Diligence llevado a cabo y un resumen con el fin de facilitar al comprador conocer los principales aspectos que deben preocuparle o centrar su atención.

- Cuerpo del Informe: se desarrolla un capítulo por cada apartado del cues- tionario preliminar. Se debe incluir una descripción de la información y datos facilitados tanto por el vendedor como por el comprador.

- Anexos: se adjuntan los anexos a los que se haya hecho referencia en el cuerpo del informe y que suelen incluir datos o documentación facilitada por el vendedor o la sociedad objeto a lo largo del ejercicio de Legal Due Diligence, cuya constatación o conocimiento directo por parte del comprador o aquel que hubiera encargado este tipo de ejercicio se considere relevante.

\section{Confidencialidad de la información}

Se debe tener presente que el método del Legal Due Diligence se lleva a cabo cuando se está negociando una fusión, sea por incorporación o por consolidación; pero en una etapa en donde las partes aún no han definido si esta se llevará a cabo o no; pues precisamente es a partir de los resultados que arroje el estudio, que se tomará la decisión final.

Para llevar a cabo dicho estudio, la empresa o empresas involucradas en el negocio revelan información que incluyen datos sensibles, importantes y vitales para el desarrollo de la empresa; que permiten identificar sus debilidades y áreas críticas.

En virtud de que durante dicha investigación se suministra información vital pero aún no se ha decidido si finalmente el negocio se va a llevar a cabo, se firman estrictas cláusulas de confidencialidad frente a terceros, con graves consecuencias civiles y penales contra el infractor, entre los representantes 
de las empresas involucradas, así como, por parte de los profesionales contratados para ejecutar el Legal Due Diligence.

Lo anterior con el fin de evitar que dicha información se filtre hacia terceras personas no vinculadas con el negocio, sea la competencia y clientes, entre otros; asimismo, este mecanismo de control resulta comprensible pues durante este proceso las partes brindan toda la información sensible de su empresa, y en el caso de que la fusión no se concrete, se garantiza que esta no sea utilizada en contra de la empresa.

\section{En sintesis}

A manera de síntesis los aspectos más relevantes del Legal Due Diligence se muestran en la figura 2:

FIGURA 2

\section{Legal Due Diligence}

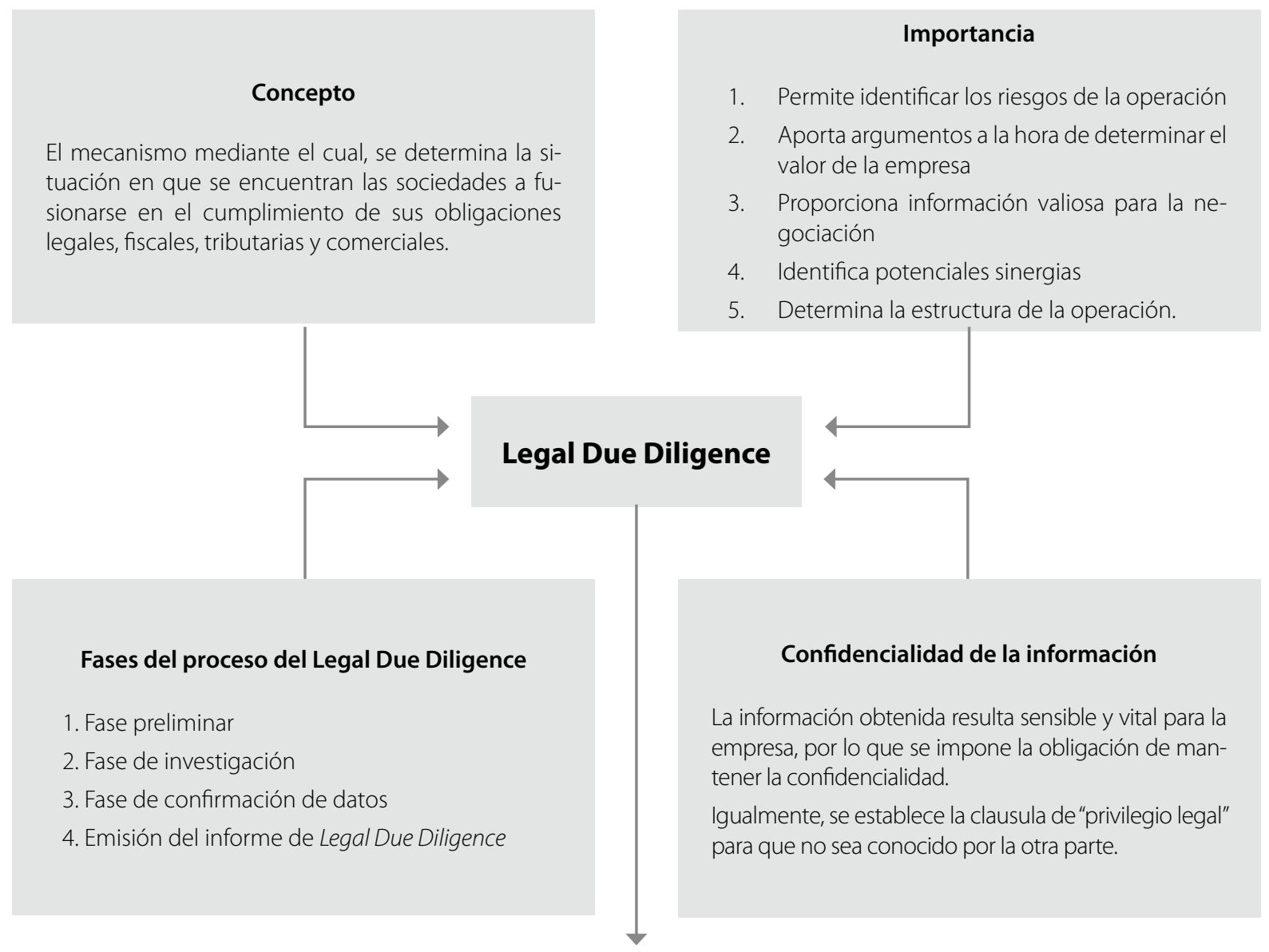

\section{Objetivo}

Conocer bien la empresa con la que se va a fusionar.

Fuente: Elaboración propia. 
Finalmente, se debe señalar que el Legal Due Diligence es un método de investigación de la información jurídica de la empresa o empresas involucradas en un proceso de fusión de sociedades anónimas, que suministra información importante para la toma de decisiones, por lo cual se recomienda su aplicación en este tipo de operaciones comerciales.

\section{Método Contable}

Desde la perspectiva contable, existen dos normas que se aplican al momento de valorar una fusión de sociedades anónimas, que son la "NIIF 3: Combinaciones de Negocios", que establece el método de compra para contabilizar la fusión; y la "NIC 7: Estados de Flujos de Efectivo" con la cual se mide la capacidad de la empresa de generar efectivo' y su equivalencia en efectivo, lo cual es importante al momento de tomar decisiones económicas, máxime en aquellas de tal relevancia como lo es una fusión.

\section{NIIF 3: Combinaciones de Negocios}

Definición y alcance

Las normas internacionales de contabilidad inicialmente y luego las normas internacionales de información financiera nacen con la finalidad de homologar los sistemas contables, a fin de unificar la información y crear un idioma común en donde ante una misma situación se utilicen los mismos conceptos contables.

1. Efectivo. Cash. Eco. Vocablo de sentido amplio asociado con cualquier transacción comercial que implique la utilización de dinero (Océano Centrum, 2001).
Con este objetivo, se aprobó la NIIF 3 denominada Combinaciones de Negocios, que precisamente se aplica en el caso de las fusiones de sociedades anónimas; en el alcance de dicha norma se señala que se aplica en los siguientes supuestos (Comité de Normas Internacionales de Contabilidad, 2009):

- Combinaciones de negocios entre entidades o negocios separados para construir un negocio conjunto.

- Combinaciones de negocios entre entidades o negocios bajo control común.

- Combinaciones de negocios en las que intervengan dos o más entidades de carácter mutualista.

- Combinaciones de negocios en las que, mediante un contrato, entidades o negocios separados se combinan para construir únicamente una entidad que informa, pero sin obtener ninguna participación en la propiedad, por ejemplo una sociedad con doble negociación en bolsa.

Esta norma establece que toda fusión de sociedades, sea por incorporación o por consolidación, debe registrarse como una compra, bajo el método de adquisición o método de compra. En el caso de la fusión por incorporación resulta sencillo identificar al comprador y registrar dentro de su información financiera dicha transacción como una compra.

Ahora bien, para las fusiones por consolidación, donde no se puede identificar claramente un comprador, se identifica a la sociedad más fuerte entre ambas, y ella será la que registre dicha operación bajo el método de adquisición. 
Bajo el método de adquisición se debe reconocer la compra de los activos, pasivos y pasivos contingentes identificables de la entidad adquirida a sus valores razonables, en la fecha de adquisición.

\section{Aplicación de la NIIF 3}

La NIIF 3 establece que las fusiones se contabilizan con el método de compra, lo cual implica que se debe identificar un comprador, en el caso de la fusión por incorporación el comprador es la empresa "sobreviviente" es decir, la que mantiene su personalidad jurídica posterior a la fusión -ver los tipos de fusión-.

Ahora bien, en la fusión por consolidación, donde desaparecen ambas, se debe identificar la que resulta más dominante para ubicarla en el papel de adquirente, ya que ella será la que registre, bajo el método de adquisición, la fusión.

Por su parte el comprador debe valuar el costo de la fusión como la suma del valor razonable a la fecha del intercambio de: (i) los activos otorgados, (ii) los pasivos incurridos o asumidos y (iii) los instrumentos de capital emitidos por el adquirente, pagados como contraprestación del control de los negocios adquiridos, más cualquier costo directamente atribuible a la combinación.

A su vez, se debe asignar el costo de la compra a la fecha de adquisición de todos los bienes del negocio a adquirir, los cuales deben ser medidos inicialmente a su valor razonable a la fecha de la adquisición.

Si existe una diferencia positiva entre el costo de la fusión y los activos, pasivos y pasivos contingentes adquiridos, se denomina crédito mercantil positivo, el cual no se amortiza y se realizan pruebas de deterioro anual o bien más frecuentemente de ser necesario.
Al contrario, si la diferencia resultare negativa, se denomina crédito mercantil negativo y se deberá reevaluar la identificación y valuación de los activos, pasivos y pasivos contingentes y en caso de persistir el importe se registrará como parte de los resultados del año.

\section{Determinación del costo de la fusión}

La determinación del costo de la fusión es un paso fundamental en el proceso de contabilización de dicha transacción, y se compone de:

a) Los valores razonables en la fecha de intercambio, los activos entregados, los pasivos incurridos o asumidos y los instrumentos de patrimonio emitidos por la adquiriente a cambio del control de la entidad adquirida.

b) Cualquier costo directamente atribuible a la combinación de negocios.

Nótese que esta norma toma como parámetro la fecha de adquisición, que es aquella en que el comprador obtiene el control efectivo sobre la entidad adquirida. Si esto se consigue mediante una única transacción de intercambio, la fecha de Intercambio coincidirá con la fecha de adquisición.

Ahora bien, puede ser necesaria más de una transacción de intercambio, por ejemplo cuando se realice por etapas, mediante compras sucesivas de acciones. En ese supuesto, el costo se determina de la siguiente manera:

a) El costo de la combinación será la suma de los costos de las transacciones individuales; $y$

b) La fecha de intercambio será la de cada una de las transacciones de intercambio (es decir, la fecha en la que cada inversión individual se reconozca en los estados financieros de la adquiriente), mientras que la fecha de adquisición será aquella en la que el comprador obtenga el control total sobre la empresa adquirida. 
Ahora bien, dentro del costo de la adquisición se deben incluir todos los gastos relacionados con la tramitación del negocio, tales como los honorarios cancelados a los especialistas, los trámites fiscales y legales, los impuestos asociados, y demás vinculados con las fases de estudio y definición de la fusión. No obstante, los gastos administrativos ordinarios como el pago de los funcionarios de planta, servicios públicos, alquileres y demás, se contabilizan como gastos normales del período, y no se incluyen dentro del costo de la fusión.

Por su parte, los costos de contratación y emisión de los pasivos financieros incluso constituirán parte integrante de la transacción de emisión de pasivos, no formarán parte del costo de la fusión, sino que se registrarán como un costo normal asociado a la operación financiera.

Igualmente, los costos de emisión de instrumentos de patrimonio que hayan sido emitidos para llevar a cabo la combinación de negocios, no forman parte del costo de la fusión, sino que se toman como un costo ordinario de la transacción de emisión de patrimonio.

\section{Distribución del costo de la fusión}

El costo de la fusión determinado previamente se distribuye en la fecha de adquisición entre los activos, pasivos y pasivos contingentes según su valor razonable, salvo en el caso de los activos no corrientes, es decir, aquellos mantenidos para su venta, como los inventarios de producto terminado, y demás, donde se reconocerán por su valor razonable menos los costos de venta correspondientes.

Para que un activo, pasivo o pasivo contingente de la empresa adquirida sea reconocido por separado por el comprador en la fecha de la adquisición, debe estar en uno de los siguientes supuestos: a) En el caso de un activo distinto de un activo intangible, si es probable que el comprador reciba los beneficios económicos futuros relacionados con el mismo, y su valor razonable se pueda medir de forma fiable;

b) En el caso de un pasivo no contingente, si es probable que la salida de recursos para liquidar la obligación incorpore beneficios económicos, su valor razonable se pueda medir de forma fiable;

c) En el caso de un activo intangible o un pasivo contingente, si sus valores razonables pueden ser medidos de forma fiable.

El comprador incorporará en su "Estado de Pérdidas y Ganancias" los resultados que genere la empresa adquirida, sea pérdida o ganancia, a la fecha de adquisición, mediante la inclusión de los ingresos y gastos de esta, basados en el costo de la fusión para el comprador. Por ejemplo, el gasto por depreciación de los activos amortizables de la empresa adquirida que, tras la fecha de adquisición, se incluirá en el "Estado de Resultados" de la empresa compradora, se basará en los valores razonables de dichos activos amortizables en la fecha de adquisición, es decir, su costo para la entidad adquiriente.

\section{Aspectos relevantes de la NIIF 3}

Finalmente, se debe señalar que la norma estudiada establece los lineamientos que se deben aplicar al momento de contabilizar una fusión de sociedades, ahora, los aspectos que resultan relevantes en dicha norma se pueden sintetizar en la Figura 3. 
FigURA 3

\section{Esquema de la NIIF 3}

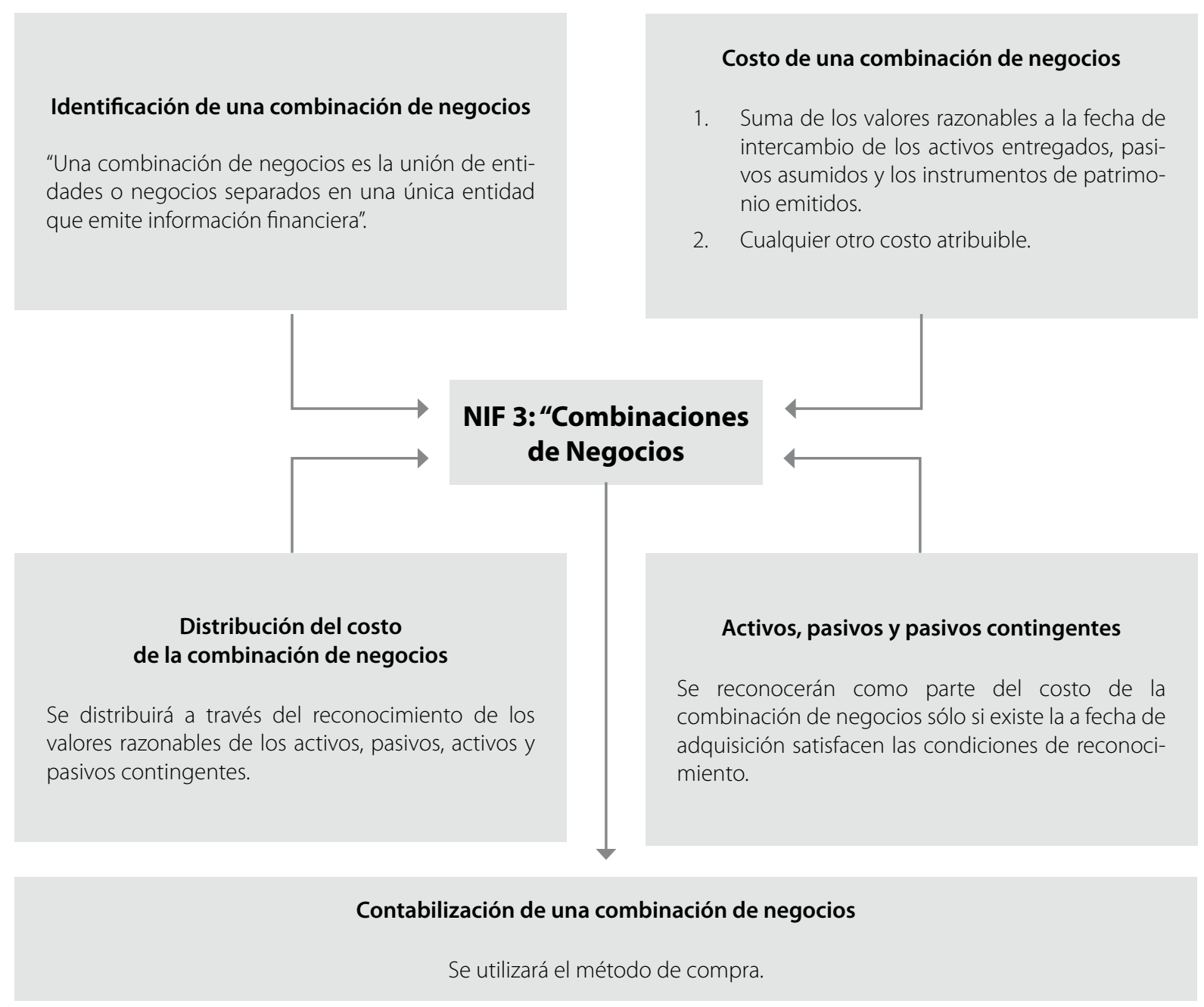

Fuente: Elaboración propia.

\section{NIC 7: Estado de Flujos de Efectivo}

\section{Concepto y alcance}

El "Estado de Flujos de Efectivo" permite evaluar la capacidad que tiene la empresa para generar efectivo y equivalentes al efectivo, así como las necesidades de liquidez, lo cual es fundamental al momento de tomar decisiones económicas y en especial en la contabilización de una fusión de sociedades.
Por "efectivo" se entiende tanto la caja ${ }^{2}$ como los depósitos bancarios a la vista; asimismo, los equivalentes al efectivo son inversiones a corto plazo de gran liquidez, que son fácilmente convertibles en importes determinados de efectivo, estando sujetos a un riesgo no significativo de cambios en su valor.

2. Caja. Cash. 1. CON. Registro en el que constan las entradas y salidas de dinero en efectivo, cuyo saldo normalmente es deudor por tratarse de una cuenta de activo (Oceano Centrum, 2001). 
La información del "Estado de Flujos de Efectivo" debe integrarse por los flujos de efectivo habidos durante el período, clasificados en: actividades de operación, actividades de inversión y actividades de financiamiento.

Ahora bien, el "Estado de Flujos de Efectivo" es uno de los más importantes para generar información económico-financiera, en la cual se basa la toma de decisiones empresariales, y por lo tanto tiene múltiples aplicaciones. No obstante, en el caso que nos ocupa se abordará la regulación de la NIC 7 en las combinaciones de negocios.

\section{Estado de Flujo de Efectivo de una fusión}

La NIC 7 establece que los flujos de efectivo derivados de combinaciones de negocios, deben ser presentados por separado, identificando claramente las empresas participantes, y clasificados como actividades de inversión.

La empresa compradora debe revelar, de forma agregada, la adquisición de una nueva empresa, en este punto se debe recordar que la NIIF 3 estudiada anteriormente establecía que la contabilización de la fusión se debía hacer bajo el método de adquisición, y para ello siempre se debe identificar un comprador; por lo tanto, quien funge con este papel, debe reflejar en sus flujos de efectivo la adquisición de la empresa como una actividad de inversión.

Por su parte, el "Estado de Flujos de Efectivo", debe contener los siguientes elementos: (Comité de Normas Internacionales de Contabilidad, 2009)

i. Contraprestación total derivada de la compra.

ii. Proporción de la contraprestación anterior satisfecha o cobrada mediante efectivo o equivalentes al efectivo.

iii. Importe de efectivo y equivalentes con que contaba la subsidiaria o la empresa de otro tipo adquirida o desapropiada. iv. Importe de los activos y pasivos, distintos de efectivo y equivalentes al efectivo correspondientes a la subsidiaria o a la empresa de otro tipo adquirida o desapropiada, agrupados por cada una de las categorías principales.

Asimismo, se debe incluir de forma separada en una sola partida, de las consecuencias que, en el efectivo y equivalentes al efectivo, ha producido la fusión, junto con la información ofrecida aparte de los importes de activos y pasivos comprados o desapropiados, con lo cual se pretende distinguir estos flujos de efectivo de aquellos otros que surgen de las actividades de operación, de inversión o de financiación.

Igualmente, se debe incluir los importes agregados que se han pagado o cobrado por la compra de la empresa.

\section{Métodos de elaboración}

El "Estado de Flujos de Efectivo" admite dos métodos de elaboración que son:

Método Directo: En este método se detallan en el estado solo las partidas que han ocasionado un aumento o una disminución del efectivo y sus equivalentes; por ejemplo: ventas cobradas, otros ingresos cobrados, gastos pagados, entre otros.

Ello conlleva a explicitar detalladamente cuáles son las causas que originaron los movimientos de recursos, exponiendo las partidas que tienen relación directa con ellos, lo cual significa una ventaja expositiva.

Método Indirecto: Consiste en presentar los importes de los resultados ordinarios y extraordinarios netos del período tal como surgen de las respectivas líneas del Estado de Resultados y ajustarlos por todas aquellas partidas que han incidido en su determinación (dado el registro en base al principio del devengo), pero que no 
han generado movimientos de efectivo y sus equivalentes.

Por lo tanto, se parte de cifras que deben ser ajustadas exponiéndose en el estado, partidas que no tienen que influir con los recursos financieros. Es por eso que este método también se llama "de la conciliación".

\section{Aspectos relevantes de la norma}

Si bien esta norma es sumamente amplia e importante por aplicarse en distintos supuestos, se pueden sintetizar los elementos de la figura 3 como los más importantes para el tema de fusión de sociedades.

FIgURA 4

\section{Esquema de la NIC 7}

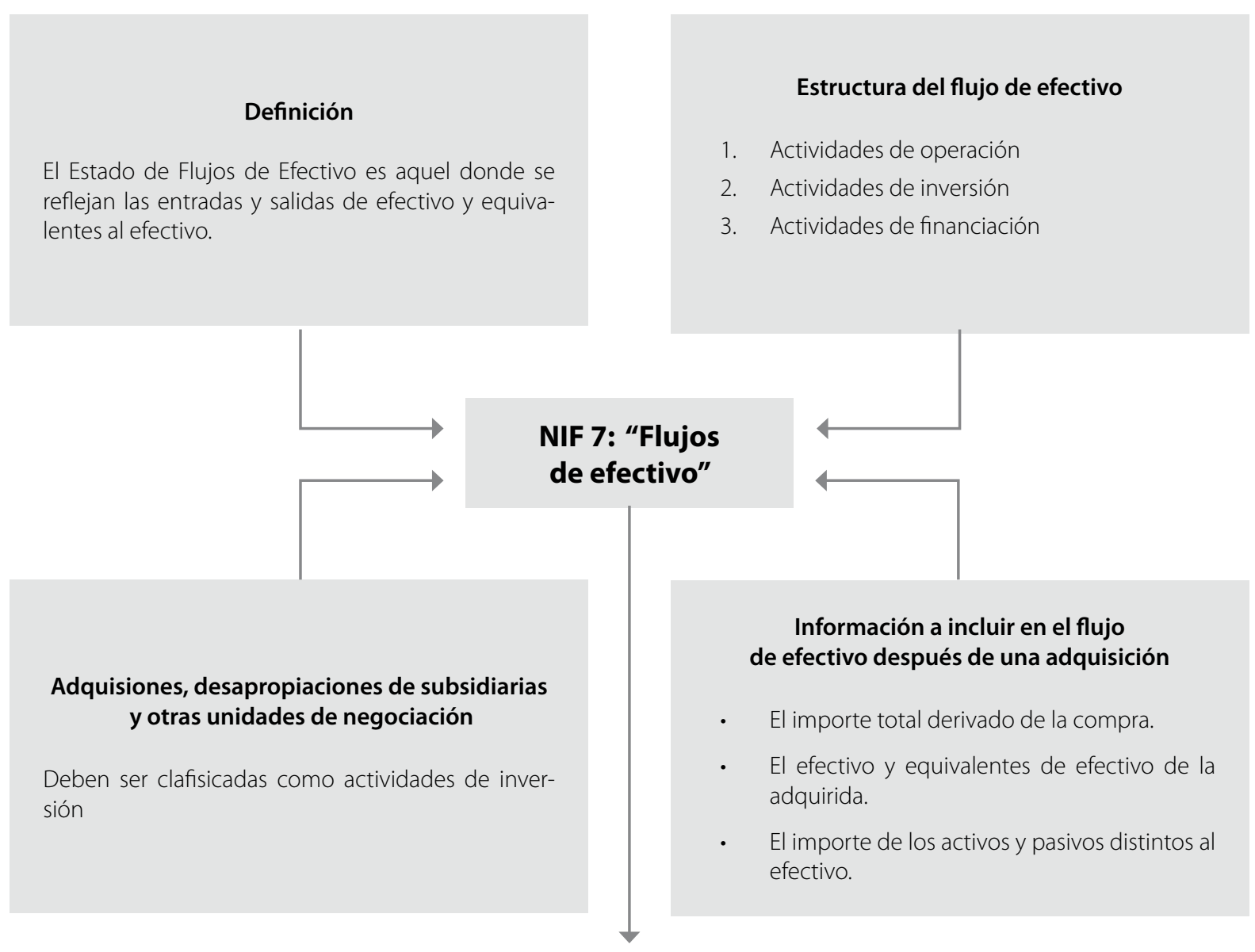

\section{Se clasifica como:}

Actividad de inversión.

Fuente: Elaboración propia. 


\section{Método Económico-Financiero: Descuento de Flujo de Caja Libre (Cash Flow)} Análisis financiero aplicado en la valoración de empresas

El análisis financiero ${ }^{3}$ permite determinar el grado de cumplimiento de las metas y planes y el desempeño de la empresa, lo cual constituye información importante al momento de decidir sobre la realización de operaciones comerciales con dicha empresa.

Ahora bien, interesa distinguir entre valor y precio, pues cuando se trata el tema de valoración de empresas, se hace referencia al intento por encontrar un valor razonable para una entidad económica dadas las condiciones económica-financieras, e incluso de mercado, en que se encuentra; mientras que el precio se refiere a la suma de dinero pactada entre comprador y vendedor por la empresa, lo cual no necesariamente coincide.

Para determinar el valor de la empresa, en el campo de las finanzas corporativas se han diseñado diversos métodos financieros como se presentan en la Tabla 2.

TABLA 2

\section{Métodos financieros aplicados para valorar empresas}

\begin{tabular}{|c|c|c|c|c|c|}
\hline $\begin{array}{l}\text { Métodos } \\
\text { basados en el } \\
\text { descuento de } \\
\text { flujos de fondos }\end{array}$ & $\begin{array}{l}\text { Métodos } \\
\text { simplificados }\end{array}$ & $\begin{array}{l}\text { Métodos basa- } \\
\text { dos en el valor } \\
\text { patrimonial }\end{array}$ & $\begin{array}{l}\text { Métodos basa- } \\
\text { dos en el benefi- } \\
\text { cio o dividendos }\end{array}$ & $\begin{array}{l}\text { Métodos basados en } \\
\text { el fondo de comercio } \\
\text { o Goodwill }\end{array}$ & Otros métodos \\
\hline $\begin{array}{l}\text { Flujo fondos } \\
\text { libre (Cash Flow) }\end{array}$ & $\begin{array}{l}\text { Modelo con } \\
\text { valor residual } \\
\text { nulo }\end{array}$ & Valor contable & $\begin{array}{l}\text { Valor de los } \\
\text { beneficios }\end{array}$ & $\begin{array}{l}\text { Método de valoración } \\
\text { "clásico" }\end{array}$ & $\begin{array}{l}\text { Método de la } \\
\text { Unión de Exper- } \\
\text { tos Contables } \\
\text { Europeos (UEC) }\end{array}$ \\
\hline $\begin{array}{l}\text { Flujo de Fondos } \\
\text { Disponibles } \\
\text { para el } \\
\text { accionista }\end{array}$ & $\begin{array}{l}\text { Modelo sin cre- } \\
\text { cimiento y con } \\
\text { perpetuidad }\end{array}$ & $\begin{array}{l}\text { Valor contable } \\
\text { ajustado (activo } \\
\text { real neto) }\end{array}$ & $\begin{array}{l}\text { Valor de los } \\
\text { dividendos }\end{array}$ & $\begin{array}{l}\text { Método simplificado } \\
\text { de la "renta abreviada } \\
\text { del goodwill" o méto- } \\
\text { do de la UEC simpli- } \\
\text { ficado. }\end{array}$ & $\begin{array}{l}\text { Método indirec- } \\
\text { to o método "de } \\
\text { los prácticos" }\end{array}$ \\
\hline \multirow[t]{3}{*}{$\begin{array}{l}\text { Flujo de Fondos } \\
\text { para la deuda }\end{array}$} & $\begin{array}{l}\text { Modelo de } \\
\text { crecimiento } \\
\text { constante y con } \\
\text { perpetuidad }\end{array}$ & $\begin{array}{l}\text { Valor de } \\
\text { liquidación }\end{array}$ & & & $\begin{array}{l}\text { Método anglo- } \\
\text { sajón o método } \\
\text { directo }\end{array}$ \\
\hline & & Valor sustancial & & & $\begin{array}{l}\text { Método de com- } \\
\text { pra de resulta- } \\
\text { dos anuales }\end{array}$ \\
\hline & & & & & $\begin{array}{l}\text { Método de tasa } \\
\text { con riesgo y de } \\
\text { la tasa sin riesgo }\end{array}$ \\
\hline
\end{tabular}

Fuente: Elaboración propia.

3. Análisis financiero. Financial analysis. Estudio de la situación financiera de una empresa para controlar los flujos de fondos, productos y servicios, tanto internos como externos de dicha empresa. 
Ahora bien, de los métodos anteriores, los más conocidos son los métodos basados en el descuento de flujos de fondos, entre ellos, el Flujo de Caja Libre (Cash Flow), y que por sus cualidad se considera como el más adecuado para efectos de una fusión, y se recomienda su utilización en dichos términos.

\section{Métodos basados en el descuento de Flujos de Fondos}

Los métodos basados en el descuento de flujos de fondos se basan en estimar el valor de la empresa en función de la proyección de cifras de las distintas variables futuras en función de las hipótesis realizadas para determinar los flujos de caja libres.

Para determinar los flujos de fondos libres futuros es necesario realizar una previsión del dinero que se obtendrá y que se deberá pagar en cada período, los cuales deben ser descontados a una tasa de descuento apropiada, según el riesgo de dichos flujos.

Todos estos métodos, parten de la siguiente expresión:

\section{$\mathrm{V}=\frac{\mathrm{CF}_{1}}{1+\mathrm{k}}+\frac{\mathrm{CF}_{2}}{(1+\mathrm{k})^{2}}+\frac{\mathrm{CF}_{3}}{(1+\mathrm{k})^{3}}+\ldots . .+\frac{\mathrm{CF}_{\mathrm{n}}+\mathrm{V}_{\mathrm{n}}}{(1+\mathrm{k})^{\mathrm{n}}}$}

En donde:

$\mathrm{CFi}=$ flujo de fondos generado por la empresa en el período i

$V n=$ valor residual de la empresa en el año $n$

$\mathrm{K}$ = tasa de descuento $\mathrm{o}$ actualización apropiada para el riesgo de los flujos de fondos.

Dichas tasas de descuento, varían según el método utilizado (Tabla 3):
TABLA 3

Tasas de Descuento según el tipo de flujo de fondos

\begin{tabular}{|l|l|}
\hline \multicolumn{1}{|c|}{ Flujos de Fondos } & \multicolumn{1}{|c|}{$\begin{array}{c}\text { Tasas de Descuento } \\
\text { Apropiadas }\end{array}$} \\
\hline Flujo de Fondos Libre & $\begin{array}{l}\text { Costo ponderado de } \\
\text { deuda (Kd) y capital (ke) }\end{array}$ \\
\hline $\begin{array}{l}\text { Flujo de Fondos } \\
\text { disponible para } \\
\text { el accionista }\end{array}$ & $\begin{array}{l}\text { Costo del capital. } \\
\text { Rentabilidad exigida } \\
\text { a las acciones (Ke) }\end{array}$ \\
\hline $\begin{array}{l}\text { Flujo de Fondos } \\
\text { para la Deuda }\end{array}$ & Costo de la Deuda (Kd) \\
\hline
\end{tabular}

Fuente: elaboración propia.

El flujo de fondos libre permite obtener el valor total de la empresa (VE). El flujo de fondos disponibles para el accionista y los dividendos permiten obtener el valor de mercado de las acciones, que unido al valor de mercado de la deuda, permitirán también establecer el valor total de la empresa.

\section{Flujo de Caja Libre (Cash Flow)}

El Flujo de Caja Libre es el flujo de fondos operativos, es decir, los fondos generados por las operaciones después de impuesto; el monto disponible después de haber cubierto las necesidades de reinversión en activos fijos y en necesidades operativas, omitiendo las deudas y cargas financieras. Supone el dinero disponible para todos los suministros de fondos de la empresa, sea de capital (accionistas) o deuda, en sus diferentes tipos.

Para calcular los flujos de fondos libres futuros se debe hacer una previsión del dinero que se recibirá y que se debe pagar en cada uno de los períodos, tal y como se hace al momento de elaborar un presupuesto de caja o presupuesto de tesorería, el cual incluye todos los movimientos 
de efectivo que pudieren darse. No obstante, la diferencia entre el Cash Flow y el presupuesto de caja o tesorería, es que en el primero el pronóstico de flujo de fondos debe realizarse sobre un periodo mucho mayor, que incluso podría ser de varios años comerciales.

Ahora bien, dichos datos no son fácilmente suministrados por los sistemas de información contables que comúnmente poseen las empresas, ya que la contabilidad financiera se basa en el principio del devengo, que se enfoca en los gastos y costos de producción; mientras que el Flujo de Caja Libre se basa en el uso del efectivo; es decir, en el dinero efectivamente recibido o entregado por la empresa.
De esta manera, para poder aplicar este método, como primer paso, se calcula el capital de trabajo sin incluir el efectivo y las inversiones temporales, suponiendo que en los Estados Financieros no se reparte todo sino que los excedentes se dejan en caja o se hacen inversiones temporales. Bajo este panorama, se calcula el flujo de caja libre, a partir del Estado de Resultados.

Como segundo paso, se vuelven a realizar los cálculos anteriores pero suponiendo que en los Estados Financieros se reparte todo el excedente de liquidez, y por lo tanto no se deja nada en caja ni se hacen inversiones temporales, situación inversa a la calculada en el primer paso. De

FIGURA 5

\section{Esquema del Flujo de Caja Libre}

\section{Flujo de caja libre (Cash Flow)}

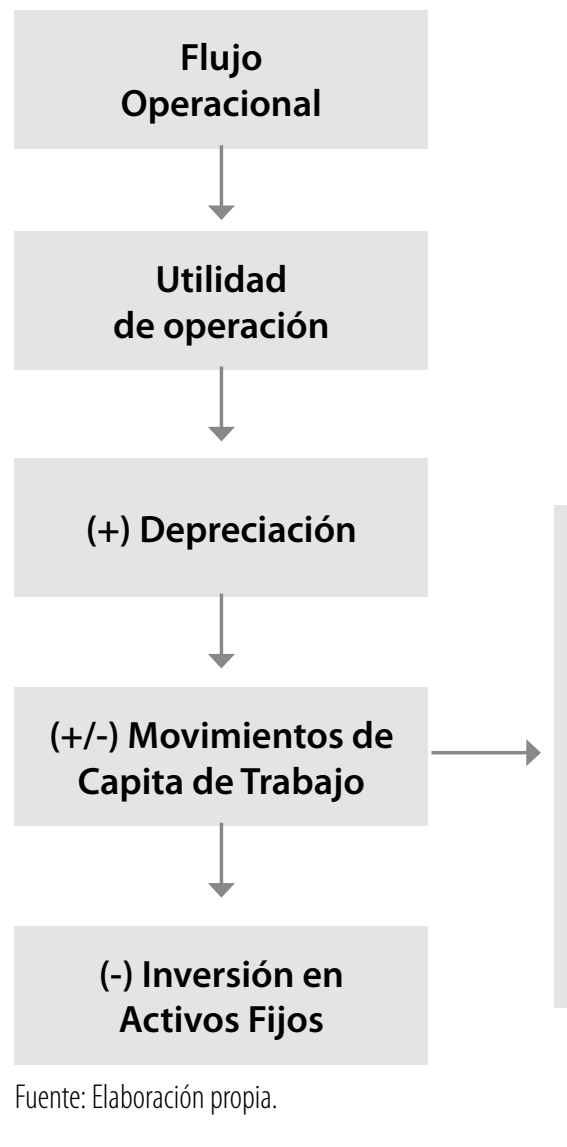

Variaciones en efectivo

- Efectivo

- Cuentas por cobrar

- Inventarios

- Gastos pagados por anticipado

- Pasivos para capital de trabajo

- Pasivos laborales

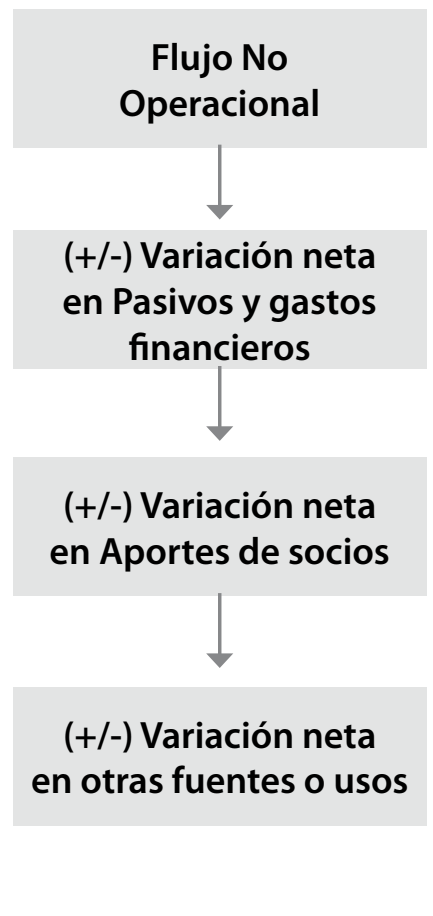


igual forma, se calcula el flujo de caja libre a partir del Estado de Resultados.

Ahora bien, este método puede sintezarse como se muestra en la Figura 5.

\section{CONCLUSIONES}

De la investigación planteada se puede concluir que la fusión de sociedades anónimas es una estrategia válida en el mundo de los negocios, y por su relevancia se encuentra tutelada por el ordenamiento jurídico costarricense.

Asimismo, precisamente por la importancia y el impacto que este tipo de negocios poseen sobre las empresas participantes se debe estudiar con exactitud todas las áreas que convergen en ella, tales como la jurídica, la contable y la económico-financiera, de tal manera que solo por medio de una correcta integración de ellas se puede tomar decisiones adecuadas y llevar a cabo una fusión de sociedades de una manera exitosa.

En el campo jurídico, es necesario realizar un análisis exhaustivo de todos los aspectos legales involucrados, de las distintas ramas del derecho, tales como: laboral, tributario, comercial, propiedad intelectual, administrativo, y demás aspectos relevantes para una fusión. Para lo cual, se recomienda la implementación del Legal Due Diligence, como el método más completo, pues constituye una guía que permite abordar todos los aspectos señalados anteriormente.

En el ámbito contable, la Normas Internacional de Información Financiera, "NIIF 3 Combinaciones de Negocios" establece que toda fusión de sociedades debe contabilizarse bajo el método de adquisición o método de compra, de tal manera que para todos los casos se debe identificar un comprador, quien deberá revelar dentro de su información financiera la compra de los activos, pasivos y pasivos contingentes de la empresa adquirida a la fecha de adquisición.
Igualmente, debe aplicarse la "NIC 7 Estado de Flujos de Efectivo", pues dicha norma constituye una guía para realizar adecuadamente el Flujo de Efectivo necesario para registrar la compra de la otra sociedad, tal y como lo establece la NIIF 3, como inversión en la información financiera de la empresa adquirente, o compradora.

Para el análisis económico-financiero, se recomienda la aplicación del método de Flujo de Caja Libre o Cash Flow, ya que este permite determinar los fondos generados por la empresa después del pago de los impuestos y las necesidades de reinversión en activos fijos y necesidades operativas de la empresa.

Finalmente, de lo expuesto se puede concluir que, a través de la integración de las áreas de convergencia jurídica, contable y económicofinanciera, por medio del Legal Due Diligencia, la "NIIF 3 Combinaciones de Negocios", la "NIC 7 Estado de Flujos de Efectivo" y el Flujo de Caja Libre o Cash Flow, se puede generar la información necesaria para que las empresas que participan en una fusión de sociedades anónimas puedan tomar decisiones con un nivel de seguridad razonable de la factibilidad y rentabilidad del negocio y a su vez minimicen los riesgos de un posible fracaso de la fusión.

\section{REFERENCIAS}

Ministerio de Justicia y Paz, Gobierno de Costa Rica. Sentencia 149-01 (Tribunal Contencioso Administrativo 25 de mayo de 2001).

Asamblea Legislativa . (1964). Código de Comercio. San José, Costa Rica.

Comité de Normas Internacionales de Contabilidad. (2009). Norma Internacional de Información Financiera 3 Combinaciones de Negocios. En Normas Internacionales de Información Financiera (págs. A93-A138).

Comité de Normas Internacionales de Contabilidad. (2009). Norma Internacional de Contabilidad 7 Estado de Flujos de Efectivo. En Normas Internacionales de Información Financiera (págs. A335-A348). 
RNA Revista Nacional de Administración

Instituto Mexicano de Contadores Públicos (2001). Boletín 8-Estados financieros consolidados y valuación de inversiones permanentes en acciones. Mexico: Departamento de Publicaciones del Instituto Mexicano de Contadores Públicos

López, M. L. (2006). La Auditoria Administrativa para evaluar el nivel de eficiencia de una empresa Administradora de Condóminos "El caso de la empresa Asesoría Plaza Guasave, SA de CV". México: Universidad Autónoma de México.

Oceano Centrum. (2001). Diccionario de Administración y Finanzas. Barcelona, España: Editorial Oceano.

Tajada, M. (2011). La Due Diligence Legal. THEMIS 59, Revista de Derecho, 213-227.

Recibido: 25-05-2011

Aceptado: 04-11-2011 